\title{
Building a better nanopore
}

\author{
Sophisticated nanopores, which utilize electron tunnelling measurements, two-dimensional materials, \\ or concepts from molecular self-assembly, could have applications in DNA and protein sequencing; the \\ technical problems that must be solved to realize such technologies are considerable though.
}

The basis of nanopore sequencing is disarmingly simple: a single strand of DNA is threaded through a nanoscale hole in a membrane with the help of an applied voltage, and modulations in an ionic current flowing through the pore are used to read the individual bases of the translocating DNA molecule. The approach does not require labels or amplification, and can be scaled up for high-throughput sequencing. Turning this concept into a practical technology was, of course, never going to be easy, but around 20 years after the idea was first proposed ${ }^{1,2}$, Oxford Nanopore Technologies now offer a commercial nanopore sequencer - the MinION. This portable device relies on protein nanopores, which are combined with processive enzymes that control the rate at which the DNA travels through the pores, and although issues over sequencing accuracy remain, performance is improving rapidly ${ }^{3}$.

Nanopores can also be made from synthetic materials, and these could, in fact, provide cheaper and more versatile devices. At present, synthetic nanopores, and related solid-state sequencing devices, are less sophisticated and capable than their biological counterparts. However, they allow read-out mechanisms other than ion-current measurements to be exploited and could, in the future, find applications in the analysis of DNA, proteins and beyond. In this issue of Nature Nanotechnology, we examine some of these possibilities in a focus on advanced nanopores.

\section{Resolution can be improved by instead detecting bases in a transverse direction and, in particular, by measuring an electron tunnelling current across a DNA molecule.}

The thickness of a solid-state nanopore is typically large compared with the size of a DNA base, which limits the resolution of measurements based on ion currents passing through the pore. Resolution can

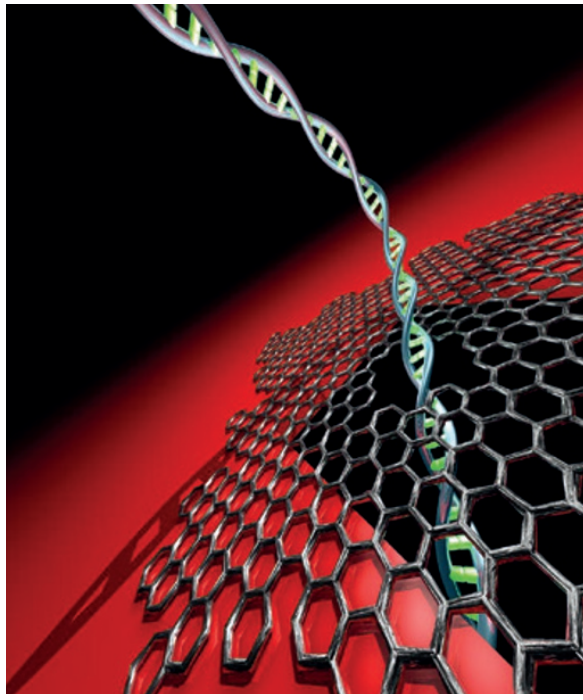

single-base resolution using ionic current measurements, the material is electrically conducting, which means that in-plane current measurements are a possibility. In a Review on page 127, Stephanie Heerema and Cees Dekker examine the use of such graphene nanodevices for DNA sequencing, highlighting the advantages, and problems, of the different approaches, which include DNA molecules passing through graphene nanopores, nanogaps, and nanoribbons, as well as the physisorption of DNA on graphene nanostructures.

The capabilities and applications of nanopore sensors can also be extended by merging them with other key areas of nanotechnology. In a Commentary on page 106, Ulrich Keyser explores the potential of combining solid-state nanopore sensing and DNA nanotechnology. DNA self-assembly methods such as DNA

be improved by instead detecting bases in a transverse direction and, in particular, by measuring an electron tunnelling current across a DNA molecule using electrodes that are separated by a small gap. This idea was first proposed in $2005^{4,5}$ and has developed quickly: proof-of-principle experiments have been reported in which single bases were differentiated in short sequences. Notably, the approach has also been used to identify individual amino acids and partially sequence peptides, suggesting it could provide a route to singlemolecule protein sequencing. In a Review on page 117, Massimiliano Di Ventra and Masateru Taniguchi consider the theoretical background to this method and the different experimental approaches that have emerged. And, in a Commentary on page 109, Stuart Lindsay explores the significant engineering challenges involved in delivering actual sequencing devices based on electron tunnelling.

Another approach to improve the performance of solid-state nanopore sensors is to make them thinner, and graphene, with its atomic thickness, is of particular interest. In the last few years, a range of graphenebased DNA sequencers have been suggested and early experimental demonstrations have begun to appear. Though it is not clear whether graphene nanopores can provide origami currently allow complex 2D and $3 \mathrm{D}$ nanostructures to be built. As Keyser explains, these methods can also enhance the specificity and sensitivity of solid-state nanopores, creating opportunities in, for example, quantitative protein analysis. (The benefits of combining nanopores and DNA nanotechnology are also highlighted in this issue on page 152, where Stefan Howorka and colleagues report using DNA to build synthetic channels that can control the transport of charged molecular cargo across a biological membrane.)

Compared with biological nanopores, synthetic nanopores still have some catching up to do. The idea of sequencing DNA using modulations in an ion current flowing through a protein nanopore has had a 10 -year head start on sequencing with electron tunnelling - in another 10, things may look quite different.

\footnotetext{
References

1. Church, G., Deamer, D. W., Branton, D., Baldarelli, R. \& Kasianowicz, J. Characterization of individual polymer molecules based on monomer-interface interactions. US patent 5,795,782 (1998).

2. Kasianowicz, J. J., Brandin, E., Branton, D. \& Deamer, D. W. Proc. Natl Acad. Sci. USA 93, 13770-13773 (1996)

3. Jain, M. et al. Nature Methods 12, 351-356 (2015).

4. Lee, J. W. \& Thundat, T. DNA and RNA sequencing by nanoscale reading through programmable electrophoresis and nanoelectrode-gated tunneling and dielectric detection. US patent 6,905,586 (2005).

5. Zwolak, M. \& Di Ventra, M. Nano Lett. 5, 421-424 (2005).
} 